\title{
25-Hydroxylase vitamin D deficiency in 27 Saudi Arabian subjects: a clinical and molecular report on CYP2R1 mutations
}

\author{
Sarah Bakhamis ${ }^{1}$, Faiqa Imtiaz², Khushnooda Ramzan², Edward De Vol ${ }^{3}$, Osamah Al-Sagheir ${ }^{4}$, \\ Abdulrahman Al-Rajhi' ${ }^{5}$, Abdullah Alashwal ${ }^{1}$, Bassam Bin Abbas ${ }^{1}$, Nadia Sakati ${ }^{1}$ and Afaf Al-Sagheir ${ }^{1}$ \\ 1Department of Pediatrics, King Faisal Specialist Hospital \& Research Centre, Riyadh, Saudi Arabia \\ ${ }^{2}$ Centre for Genomic Medicine, King Faisal Specialist Hospital \& Research Centre, Riyadh, Saudi Arabia \\ ${ }^{3}$ Department of Biostatistics, Epidemiology \& Scientific Computing, King Faisal Specialist Hospital \& Research Centre, Riyadh, Saudi Arabia \\ ${ }^{4}$ Department of Medicine, King Faisal Specialist Hospital \& Research Centre, Riyadh, Saudi Arabia \\ ${ }^{5}$ Department of Orthopedics, King Saud University Medical City, Riyadh, Saudi Arabia
}

Correspondence should be addressed to A AlSagheir: asagheir@kfshrc.edu.sa

\begin{abstract}
Vitamin D deficiency remains a major cause of rickets worldwide. Nutritional factors are the major cause and less commonly, inheritance causes. Recently, CYP2R1 has been reported as a major factor for 25-hydroxylation contributing to the inherited forms of vitamin D deficiency. We conducted a prospective cohort study at King Faisal Specialist Hospital \& Research Centre, Riyadh, Saudi Arabia, to review cases with 25-hydroxylase deficiency and describe their clinical, biochemical, and molecular genetic features. We analyzed 27 patients from nine different families who presented with low 25-OH vitamin $\mathrm{D}$ and not responding to usual treatment. Genetic testing identified two mutations: c.367+1G>A (12/27 patients) and c.768dupT (15/27 patients), where 18 patients were homozygous for their identified mutation and 9 patients were heterozygous. Both groups had similar clinical manifestations ranging in severity, but none of the patients with the heterozygous mutation had hypocalcemic manifestations. Thirteen out of 18 homozygous patients and all the heterozygous patients responded to high doses of vitamin D treatment, but they regressed after decreasing the dose, requiring lifelong therapy. Five out of 18 homozygous patients required calcitriol to improve their biochemical data, whereas none of the heterozygous patients and patients who carried the c.367+1G $>$ A mutation required calcitriol treatment. To date, this is the largest cohort series analyzing CYP2R1-related 25-hydroxylase deficiency worldwide, supporting its major role in 25-hydroxylation of vitamin D. It is suggested that a higher percentage of CYP2R1 mutations might be found in the Saudi population. We believe that our study will help in the diagnosis, treatment, and prevention of similar cases in the future.
\end{abstract}

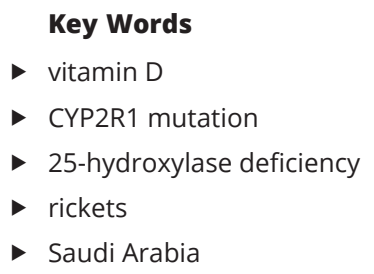

Endocrine Connections (2021) 10, 767-775

\section{Introduction}

Vitamin D deficiency and associated rickets are global issues in the younger age group. Although nutritional factors and inadequate sunlight exposure remain the most common causes of rickets worldwide, more recently, the heritable and genetic causes of rickets have become a major factor (1). Despite abundant exposure to sunshine in Saudi Arabia, rickets remains predominant and a major public health problem in the population (2). A crosssectional study of 2110 Saudi children and adolescents showed an overall prevalence of $95.3 \%$ with combined https://ec.bioscientifica.com https://doi.org/10.1530/EC-21-0102 (c) 2021 The authors Published by Bioscientifica Ltd
This work is licensed under a Creative Commons Attribution-NonCommercial-NoDerivatives 4.0 Internationab bicense ifica.com at 04/26/2023 09:38:37Am 
vitamin D deficiency and insufficiency (3). Genetic and hereditary factors might contribute to these results, as consanguinity levels are high in the Saudi population, reaching up to $56 \%$ (4).

Vitamin D is a fat-soluble vitamin that reaches its active form 1,25-(OH)2 $\mathrm{D} 3$ through a complex biosynthesis pathway involving two steps of hydroxylation: first by 25 -hydroxylase in the liver, followed by $1 \alpha$-hydroxylase in the kidney (5). A mutation in the CYP27B1 gene, which catalyzes the $1 \alpha$-hydroxylase enzyme, can prompt the inability to synthesize the active form of vitamin D $(1,25-[\mathrm{OH}] 2 \mathrm{D} 3)$, which results in vitamin D-dependent rickets type 1A (VDDR1A, MIM264700). Mutation in the VDR gene coding for the vitamin $\mathrm{D}$ receptor can lead to vitamin D-dependent rickets type 2A (VDDR2A, MIM277440). In contrast, there are no enough data about the hepatic CYP enzymes that are involved in 25-hydroxylation as a cause of genetic vitamin D deficiency $(5,6)$. Studies have demonstrated the relative relation between CYP27A1 and CYP2R1 as potent enzymes in the hydroxylation of carbon-25 of vitamin D. However, CYP2R1 appears to be the major enzyme of 25-hydroxylase due to its low capacity and high affinity toward vitamin D 25-hydroxylase, and it can hydroxylase both ergocalciferol (D2) and cholecalciferol (D3) at the 25 position, but other enzymes with 25 -hydroxylase activity may also contribute to the circulating levels of $25-\mathrm{OH}$ D3 $(6,7,8)$.

The 25-hydroxylase deficiency was initially reported in two Nigerian siblings who presented with severe bone disease associated with biochemical evidence of rickets (9). Molecular genetic analysis identified a c.296T $>C$ (p.Leu99Pro) mutation in the CYP2R1 gene $(9,10)$. Thacher and colleagues also described two additional families and a new CYP2R1 mutation, c.726A>C (p.Lys242Asn), during a further screening of the Nigerian population with rickets (11). Another two siblings from Saudi Arabia who presented with typical features of vitamin D deficiency and rickets, with normal nutritional and developmental history, were found to be compound heterozygous for two mutations (c.367+1G>A and c.768dupT) in CYP2R1 (8). Recently, Molin et al. reported two unrelated families from France and Morocco with homozygous CYP2R1 mutations. They reported a new variation c.124_138delinsCGG (p.Gly42_Leu46delinsArg) and the previously published c.296T>C (p.Leu99Pro) mutation (12). These data identify the potential relation of 25-hydroxyvitamin $\mathrm{D}$ deficiency with CYP2R1 as a major biological activator of vitamin D 25-hydroxylase in which this new entity of the disease was described as vitamin D-dependent rickets type $1 \mathrm{~B}$ (VDDR1B, MIM600081) $(8,11,12)$.
The main aim of this study was to investigate the presence of 25-hydroxylase deficiency in Saudi patients and to describe in detail their clinical, biochemical, and molecular genetic features, which we hope will provide beneficial insight into this disorder.

\section{Patients and methods}

\section{Patients}

A prospective cohort study was conducted at King Faisal Specialist Hospital \& Research Centre, Riyadh, Saudi Arabia. All patients were collected from the pediatric endocrine clinic from 1990 to 2017, who presented with classical symptoms of vitamin D deficiency and low 25-OH vitamin D levels $(<50 \mathrm{nmol} / \mathrm{L})$ despite good nutrition and sun exposure, or were asymptomatic patients with low $25-\mathrm{OH}$ vitamin $\mathrm{D}$ levels $(<50 \mathrm{nmol} / \mathrm{L})$, and were either not responding to high doses of vitamin $\mathrm{D}$ therapy (2000-5000 IU/day or 50,000 IU weekly) or were dependent on it. The patients' relatives who had similar presentations were also recruited after genetic counseling. All index patients and their family members underwent molecular genetic testing for CYP2R1 mutations and were included in this study if they were positive for the mutation. We excluded patients who had nutritional, malabsorption, or comorbidity factors (i.e. celiac disease, liver disease, or renal failure), and patients who were on anti-epileptic medications.

The medical charts of all patients were reviewed for demographic, clinical, laboratory, and radiological data. After recruiting them, all patients were started on a high dose of vitamin D therapy (50,000 IU/week for 8-12 weeks) along with an oral calcium supplement of $50 \mathrm{mg} / \mathrm{kg}$, and their response to treatment was monitored. Compliance was tracked by contacting the patients and by monitoring the patients' medication dispensers, i.m. doses were given to the patients who were not in compliance with their medication. Patients were considered as 'not responding' if their serum 25-hydroxyvitamin D (25-OH D3) did not reach at least $52 \mathrm{nmol} / \mathrm{L}$ at any time of their treatment period, with or without biochemical marker improvement (alkaline phosphatase, serum parathyroid hormone, calcium, phosphate).

Regarding ethical considerations, this study was approved by the institutional review board Research Advisory Council at King Faisal Specialist Hospital \& Research Centre (RAC \#2151206), which adheres to the institutional guidelines and regulations. All of the

This work is licensed under a Creative Commons Attribution-NonCommercial-NoDerivatives 4.0 elnternationad dicense ifica. com at 04/26/2023 09:38:37AM 
investigations and treatment plans were part of the patients' medical care, and full informed consent was taken from the patients or their guardians.

\section{Biochemical and radiological assessments}

A whole blood sample was taken from each patient without fasting at baseline and after the high-dose vitamin $\mathrm{D}$ treatment course ( 8 weeks), and during the course of the study, biochemical markers were rechecked whenever the dose was maintained or changed. Total serum 25-hydroxyvitamin $\mathrm{D}$ was measured by electrochemiluminescence binding assay used on Elecsys ${ }^{\circ}$ and cobas e 411 immunoassay analyzers (Roche Diagnostics). Based on the latest recommendation of the Endocrine Society, patients were diagnosed with vitamin D deficiency if serum 25-OH D3 level was less than $20 \mathrm{ng} / \mathrm{mL}$ (50 nmol/L), and vitamin D insufficiency if serum $25-\mathrm{OH}$ D3 level was between 21 and $29 \mathrm{ng} / \mathrm{mL}$ (52-72 nmol/L). Sufficient level was defined when serum 25-OH D3 level was 30-100 ng/mL (75-250 nmol/L) (13).

Serum parathyroid hormone was measured by electrochemiluminescence immunoassay 'ECLIA' used on Elecsys and cobas e 411 immunoassay analyzers (Roche Diagnostics), where serum level above $6.9 \mathrm{pmol} / \mathrm{L}$ (65 ng/L) was considered abnormal (14). Serum calcium, phosphorus, and alkaline phosphatase were measured by photometric assay used on Hitachi cobas c systems (Roche Diagnostics).

Plain X-rays of the patients' upper and lower extremities were reviewed, looking for signs of rickets (i.e. demineralization, widening, and cupping of the physeal regions, metaphyseal fraying, bowing and development of knock-knees, or genu-valgum).

\section{Molecular assessment}

Genomic DNA from whole blood was extracted from clinically diagnosed patients with 25-hydroxylase deficiencies by standard salt precipitation methods. Mutation analysis of the CYP2R1 gene was performed by Sanger DNA sequencing. The coding exons and exonintron boundaries of CYP2R1 were amplified by PCR using intronic primers designed using Primer3, v.0.4.0 software (http://frodo.wi.mit.edu/primer3/). Mutation screening was performed by bidirectional sequencing using the BigDye ${ }^{\bullet}$ Terminator v3.1 Cycle Sequencing Kit on an ABI PRISM 3730 DNA analyzer (Applied Biosystems). The sequence analysis was performed using the SeqMan 6.1 module of the Lasergene (DNAStar Inc., Madison, WI, USA) software package and then compared to the reference sequence database GenBank (accession number CYP2R1; NM_024514). Segregation of the identified variants within the family and analysis of 200 ethnically matched control alleles were also performed in a similar way. The sequences of primers and the PCR conditions are provided in Supplementary Table 1 (see section on supplementary materials given at the end of this article).

\section{Statistical analysis}

The nature of the data collected was primarily counted data and is presented in the form of cross-tabulations. For continuously scaled measures (e.g. 25-OH vitamin D level), means and S.D. were used to summarize the distributions. In investigating the relationships between variables used in the cross-tabulations, Chi-square tests (or Fisher's exact test in the case of two-by-two tables) were used to evaluate relationships between the two variables. For analyses involving continuously scaled data (e.g. initial 25-OH vitamin D), a Wilcoxon rank-sum test was carried out to compare those data between groups defined by mutation, zygosity, or response to treatment. Stratified two-by-two tables were also analyzed to investigate the relationship between symptoms (e.g. bone pain, short stature) and the mutation present while stratifying on zygosity (i.e. homozygous or heterozygous). Similarly, an analysis of the relationship between symptoms and zygosity while stratifying on mutation was carried out. Finally, the above two stratified analyses were also conducted with the outcomes (biochemical, radiological, and response to treatment) instead of symptoms. This stratification analysis was based on the Mantel-Haenszel method. Statistical significance was concluded when the $P$ value was less than 0.05 .

\section{Results}

A total of 27 patients from 9 different families were identified (11 males and 16 females) (Fig. 1). Their ages of presentation ranged between 2 and 10 years, and their ages at the time of the study ranged between 6 and 50 years. All were found to have a significant family history of vitamin $\mathrm{D}$ deficiency (i.e. documented vitamin $\mathrm{D}$ deficiency in more than one family member who required continuous treatment with a vitamin D supplement).

Our patients presented with variable symptoms: 25/27 had bone pain, 18 out of 27 had limitations of physical activity, and 12 out of 27 presented with short stature.

This work is licensed under a Creative Commons Attribution-NonCommercial-NoDerivatives 4.0 elfternationab ficense.ifica.com at 04/26/2023 09:38:37AM 
Family 1

c.768 ins T

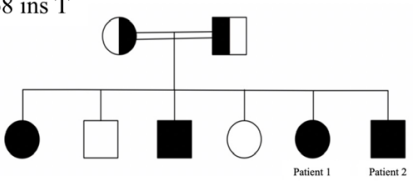

Family 2

c. $367+1 \mathrm{G}>\mathrm{A}$

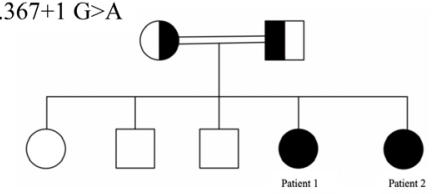

Family 3

c. $367+1 \mathrm{G}>\mathrm{A}$

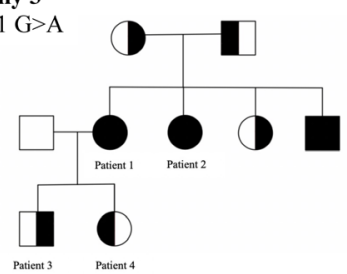

Family 4 c.768 ins T
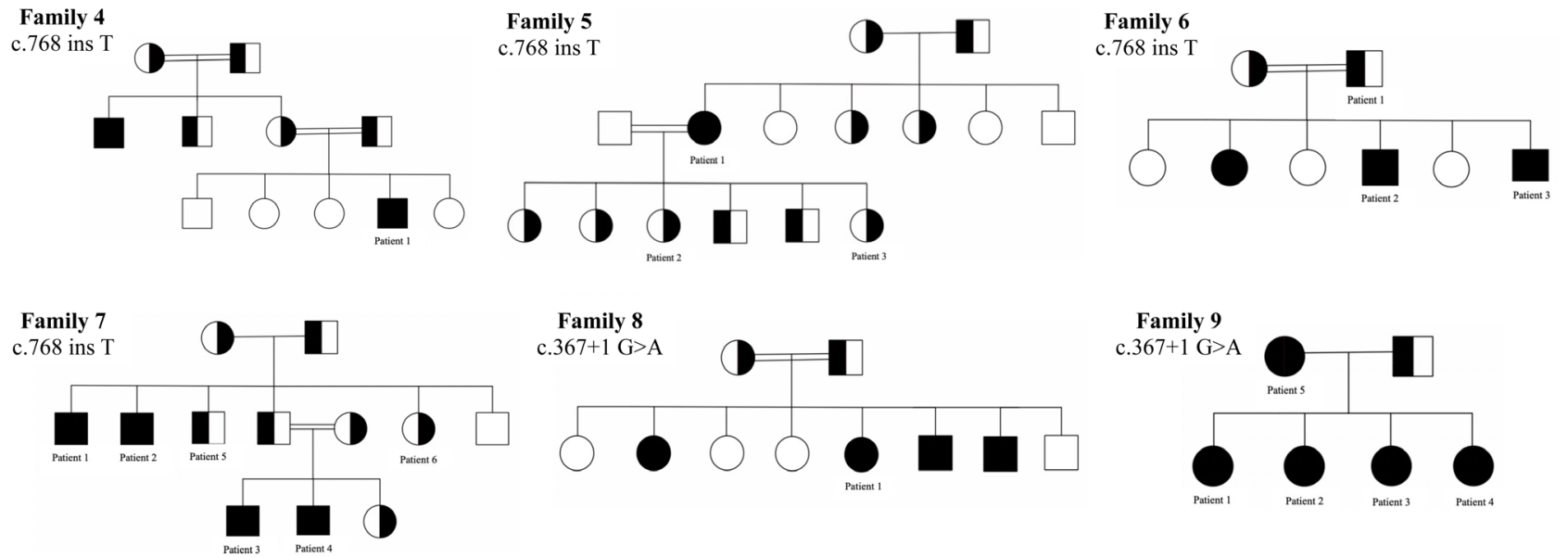

Figure 1

Family pedigrees with their corresponding genetic mutations. $\square$ : homozygous mutation, $\mathbf{0} \square$ : heterozygous mutation, $\bigcirc \square$ : normal.

Some patients had a severe presentation in the form of bone deformity (8/27) and hypocalcemic manifestations $(5 / 27)$ (i.e. seizure, carpopedal spasm, muscle cramps, and twitching). The genetic testing identified two CYP2R1 variants in our patient cohort: c.367+1G>A (12/27) and c.768dupT, p.Leu257SerfsTer6 (15/27). Eighteen patients were found to be homozygous for a specific mutation and nine patients were heterozygous for the identified mutation (Fig. 2 and Table 1). The prevalence of variant
c.768dupT is 0.0000319 and c. $367+1 \mathrm{G}>\mathrm{A}$ is $0.0000159 \mathrm{in}$ gnomAD. These variants were not found in 200 ethnically matched alleles, thus supporting that these variants are not population-specific polymorphisms. ClinVar and ACMG interpretations also provide evidence that the identified CYP2R1 variants are clinically significant and are predicted to be pathogenic (Table 2 ).

A comparison between the homozygous and heterozygous patients, clinical, biochemical, and

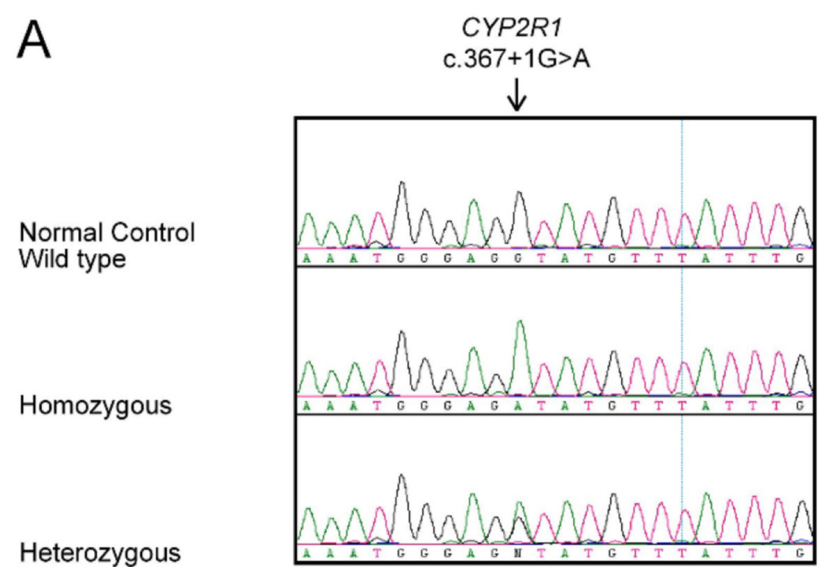

B

Normal Control Wild type

Homozygous

Heterozygous
CYP2R1

c.768dupT $\downarrow$

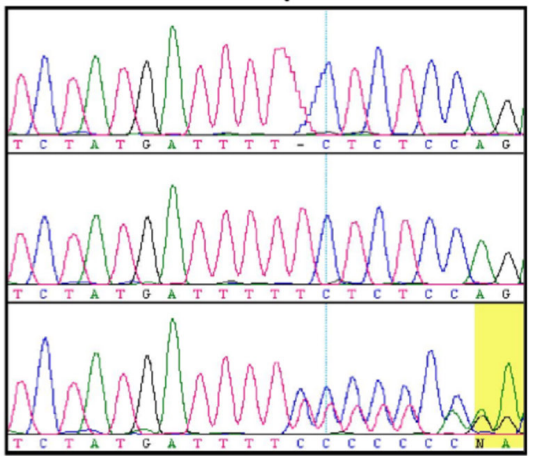

\section{Figure 2}

Sequence chromatograms of normal control, heterozygous, and homozygous individuals for the identified CYP2R1 variants (A) c.367+1G>A and (B) c.768dupT in our patients' cohort.

https://ec.bioscientifica.com https://doi.org/10.1530/EC-21-0102 (c) 2021 The authors Published by Bioscientifica Ltd

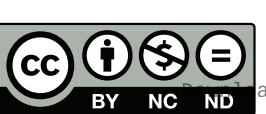

This work is licensed under a Creative Commons Attribution-NonCommercial-NoDerivatives 4.0 elfternational bicense ifica.com at 04/26/2023 09:38:37AM 
Table 1 Demographic data of all patients included in the study.

\begin{tabular}{|c|c|c|c|}
\hline & $\begin{array}{l}\text { Homozygous } \\
(n=18)\end{array}$ & $\begin{array}{l}\text { Heterozygous } \\
\qquad(n=9)\end{array}$ & $\begin{array}{l}\text { Total } \\
(n=27)\end{array}$ \\
\hline \multicolumn{4}{|l|}{ Gender } \\
\hline Male & $8 / 18$ & $3 / 9$ & $11 / 27$ \\
\hline Female & $10 / 18$ & $6 / 9$ & $16 / 27$ \\
\hline $\begin{array}{l}\text { Age of presentation, } \\
\text { years }\end{array}$ & $2-6$ & $6-10$ & Mean age 6 \\
\hline \multicolumn{4}{|l|}{ Genetic mutation } \\
\hline c. $367+1 G>A$ & $8 / 18$ & $4 / 9$ & $12 / 27$ \\
\hline c.768dupT & $10 / 18$ & $5 / 9$ & $15 / 27$ \\
\hline
\end{tabular}

radiological data are presented in Table 3 . Across the clinical features, there was no statistically significant difference in the rate of a feature between the two zygosities (homozygous or heterozygous). It is noteworthy that none of the heterozygote patients manifested hypocalcemia symptoms, compared with 4 out of 18 homozygous subjects with such manifestations $(P=0.2677)$. The nonsignificance of this differential rate may be attributed to small numbers.

When analyzing the relationships between initial 25-OH vitamin $\mathrm{D}$ and other factors, a significant relationship was found with zygosity $(P=0.0008)$ with higher initial values for heterozygote patients; nosignificant relationship was found with the type of mutation $(P=0.8755)$ (Fig. 3A and B). For the biochemical feature of the bone profile, homozygotes showed a statistically higher rate of abnormality $(P=0.0235)$. For the radiological manifestations, the rate of some type of abnormal manifestation was statistically higher $(P=0.0036)$ in the homozygote group (13/18) than in the heterozygote group (2/9). However, looking individually amongst any of the types of such manifestations (e.g. cupping, geno-valgus, rachitic rosary), no statistically significant differences could be found. Generalized osteopenia was the major radiological feature and was found to have a statistically higher rate among the homozygotes $(P=0.0036)$.

Thirteen out of 18 of the homozygous group and all of the heterozygous group responded to the treatment, but they showed regression after decreasing the vitamin D dose to the daily requirement dose and, therefore, were moved to a high vitamin D dose as maintenance. Their variable response to treatment and maintenance requirements is shown in Table 4. Although all of the heterozygote group vs 13 of the 18 homozygote group responded, this difference was not statistically significant $(P=0.1358)$. Patients' maintenance treatments followed a protocol of first monthly treatments, then progressively more frequent if there was no response. In Table 4, it can be seen that 7 out of 9 of the heterozygote patients responded to the

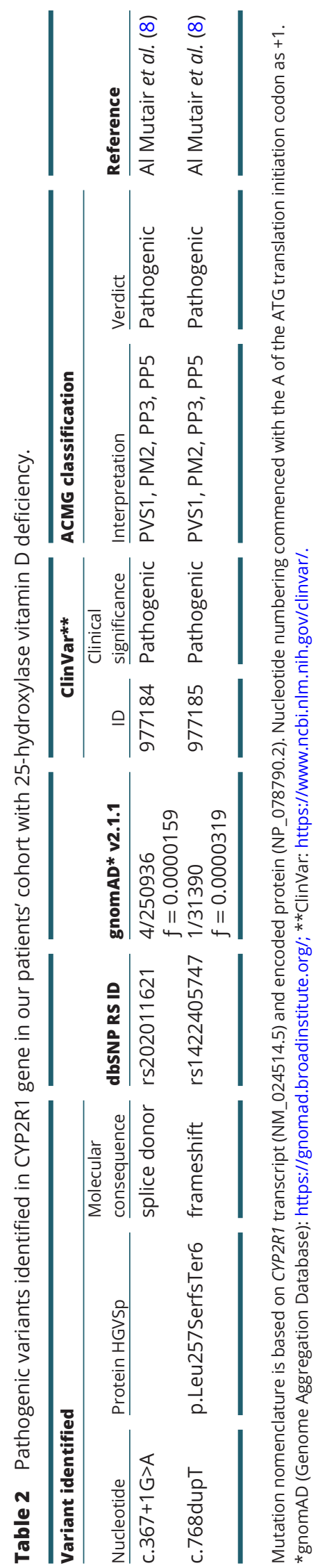

This work is licensed under a Creative Commons Attribution-NonCommercial-NoDerivatives 4.0 
Table 3 Comparison between the heterozygous and homozygous (presenting symptoms, biochemical, and radiological abnormalities).

\begin{tabular}{|c|c|c|}
\hline & Heterozygous $(n=9)$ & Homozygous $(n=18)$ \\
\hline \multicolumn{3}{|l|}{ Presenting symptoms: } \\
\hline Bone pain & $7 / 9$ & $18 / 18$ \\
\hline Short stature & $3 / 9$ & $9 / 18$ \\
\hline Limitation of activity & $5 / 9$ & $13 / 18$ \\
\hline Bone deformity & $1 / 9$ & $7 / 18$ \\
\hline Gait abnormality & $1 / 9$ & $6 / 18$ \\
\hline Hypocalcemia manifestation (Seizure, carpopedal spasm, muscle cramps, and twitching) & $0 / 9$ & $5 / 18$ \\
\hline $25-\mathrm{OH}$ vitamin D levels range, $\mathrm{nmol} / \mathrm{L}$ & $6-25$ & $<6$ \\
\hline Abnormal bone profile (high parathyroid hormone, low calcium, high alkaline phosphatase) & $4 / 9$ & $16 / 18$ \\
\hline \multicolumn{3}{|l|}{ Radiological manifestations: } \\
\hline Generalized osteopenia & $2 / 9$ & $13 / 18$ \\
\hline Cupping & $0 / 9$ & $4 / 18$ \\
\hline Geno-valgus & $1 / 9$ & $7 / 18$ \\
\hline Rachitic rosary & 0 & $1 / 18$ \\
\hline Normal & $7 / 9$ & $5 / 18$ \\
\hline
\end{tabular}

monthly protocol vs 4 out of 18 among the homozygote patients $(P=0.0115)$. When the frequency of treatment was increased to twice monthly, we were able to achieve an overall response of 8 out of $9(7 / 9+1 / 9)$ in the heterozygote group vs 7 out of $18(4 / 18+3 / 18)$ in the homozygote group $(P=0.0192)$.

Table 5 is a further extension of Tables 3 and 4, based on the identified mutation. As anticipated from the overall result, no significant differences in the clinical manifestations between heterozygote and homozygote patients were found in either of the mutation groups. With respect to the observation about hypocalcemia only occurring in the homozygote group, it is interesting to note that this manifestation was found approximately equally in both mutation groups. The stratification in Table 5 allowed us to investigate the extent to which there may be a difference in clinical manifestations between the two types of mutations while stratifying on zygosity.
These clinical differences were evaluated through a Mantel-Haenszel methodology. No differences were found, suggesting no overall differential deleterious effect of one mutation vs the other. In addition to that, the results for the biochemical markers were assessed separately for the two mutation groups. The rates of abnormal bone profile in the two groups were 10 out of 15 and 10 out of 12 patients for the c.768dupT and c.367+1G $>$ A mutation groups, respectively $(P=0.4082)$. When this was stratified by zygosity in the Mantel-Haenszel methodology, no significance was found $(P=0.2855)$. Also in Table 5 , the rates of response for the two types of mutations are given: 12 out of 12 patients with c. $367+1 \mathrm{G}>$ A mutation group and 10 out of 15 patients with c.768dupT mutation group. This difference is marginally statistically significant $(P=0.0470)$. For the homozygous subgroup alone, the difference was solidly significant $(P=0.0359)$, and after stratifying on zygosity through the Mantel-Haenszel methodology,
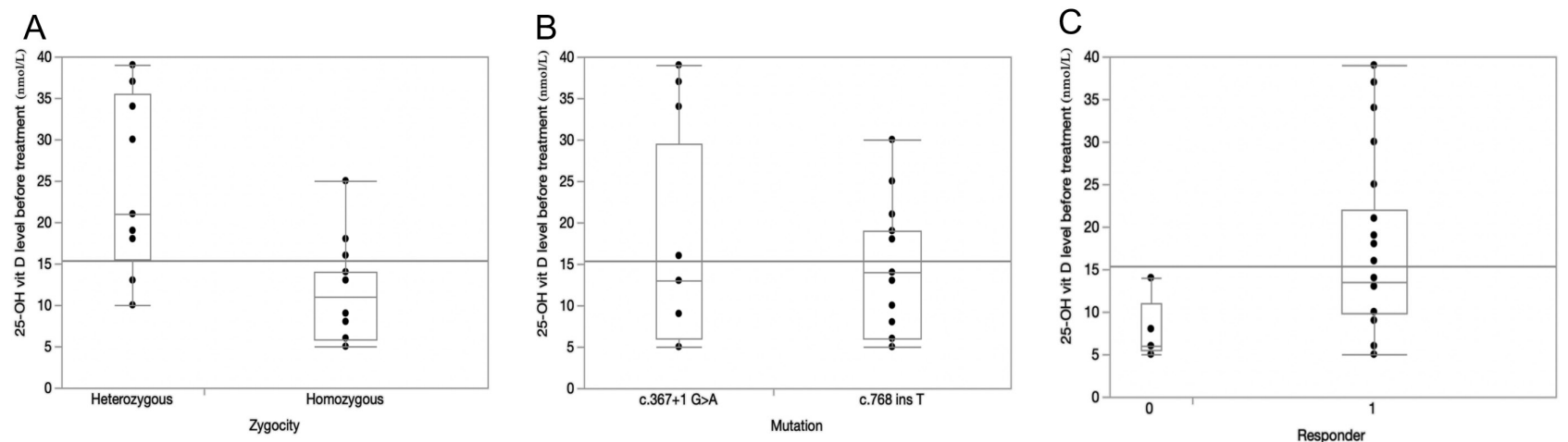

Figure 3

(A) Analysis of initial 25-OH vitamin D level by Zygosity $(P=0.0008) ;(B)$ analysis of initial $25-\mathrm{OH}$ vitamin $\mathrm{D}$ level by mutation $(P=0.8755) ;(\mathrm{C})$ analysis of initial $25-\mathrm{OH}$ vitamin $\mathrm{D}$ level and response to treatment $(P=0.0509)$.

https://ec.bioscientifica.com https://doi.org/10.1530/EC-21-0102 (c) 2021 The authors Published by Bioscientifica Ltd

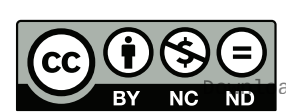

This work is licensed under a Creative Commons Attribution-NonCommercial-NoDerivatives 4.0 International ticense.ifica com at $04 / 26 / 2023 \quad 09: 38: 37$ MM 
Table 4 Comparison between the heterozygous and homozygous in their response to treatment.

\begin{tabular}{|c|c|c|}
\hline & $\begin{array}{l}\text { Heterozygous } \\
\qquad(n=9)\end{array}$ & $\begin{array}{c}\text { Homozygous } \\
(n=18)\end{array}$ \\
\hline Response to treatment & $9 / 9$ & $13 / 18$ \\
\hline \multicolumn{3}{|l|}{ Types of maintenance therapy: } \\
\hline High dose of vitamin D therapy & $9 / 9$ & $13 / 18$ \\
\hline Weekly & $1 / 9$ & $6 / 13$ \\
\hline Twice monthly & $1 / 9$ & $3 / 13$ \\
\hline Monthly & $7 / 9$ & $4 / 13$ \\
\hline Calcitriol $\left(1,25-(\mathrm{OH})_{2}\right.$ vitamin D) & $0 \% *$ & $5 / 18$ \\
\hline
\end{tabular}

*Was not tried/no need.

the difference is even more strongly significant $(P=0.0222)$. Additionally, the relationship between initial 25-OH vitamin $\mathrm{D}$ and response to treatment was investigated and a marginally significant $(P=0.0509)$ result was found, with responders tending to be patients who have higher initial levels (Fig. 3C).

\section{Discussion}

Selective 25-hydroxylase deficiency is a rare disorder that is not fully described in the literature, which might reflect the number of misdiagnosed subjects who present with vitamin $\mathrm{D}$ deficiency rickets and did not improve with regular therapy. Only six families have been reported worldwide for selective 25-OH deficiency, and five different mutations were identified in the CYP2R1 gene $(8,9,11,12)$, described as vitamin D-dependent rickets type 1B (VDDR1B, MIM600081). In our study, we analyzed 27 patients from 9 different families with mutations in CYP2R1, which is the largest cohort of VDDR1B to date. With this large number of patients, we had the chance to elaborate more on the clinical presentation, variability of the disease, and response to treatment.

All patients described in our study presented with classical clinical, biochemical, and radiological features of vitamin D deficiency and associated rickets. They were treated with a high dose of vitamin D therapy (50,000 IU/ week for 8-12 weeks), which resulted in the resolution of biochemical abnormalities and radiographic deformities in some of them. The fact that their 25-OH D3 levels dropped after lowering the dose to normal daily requirement raised the suspicion of an underlying enzymatic defect, which was confirmed by having selective 25-hydroxylase deficiency by a molecular study of CYP2R1 that revealed either one of the two mutations (c.768dupT and c.367+1 G>A). The two identified mutations found in our patients had been previously reported by Al Mutair et al. in the Saudi siblings (c.768dupT and c.367+1G>A), which might reflect the genetic background of the disease in the Arab region (8).

In previous studies, treatment with supra-therapeutic doses of oral vitamin $\mathrm{D}$ in addition to oral calcium showed minimal to moderate clinical and biochemical response depending on their homozygous/heterozygous status and the underlying genetic mutation, in which homozygous patients showed notably lessened response compared with heterozygous patients. Even though heterozygous patients had a better response, they were unable to achieve an optimal level of $25-\mathrm{OH}$ vitamin $\mathrm{D}(8,11)$.

Table 5 Comparison between the two identified mutations (c.367+1G>A and c.768dupT) (clinical presentation and response to treatment).

\begin{tabular}{|c|c|c|c|c|}
\hline & \multicolumn{2}{|c|}{ c.768dupt $(n=15)$} & \multicolumn{2}{|c|}{$\mathbf{c . 3 6 7 + 1 G}>\mathbf{A}(n=12)$} \\
\hline & Homozygous $(n=10)$ & Heterozygous $(n=5)$ & Homozygous $(n=8)$ & Heterozygous $(n=4)$ \\
\hline \multicolumn{5}{|l|}{ Clinical presentation: } \\
\hline Bone pain & $9 / 10$ & $3 / 5$ & $7 / 8$ & $4 / 4$ \\
\hline Short stature & $4 / 10$ & $2 / 5$ & $5 / 8$ & $1 / 4$ \\
\hline Limitation of activity & $6 / 10$ & $4 / 5$ & $7 / 8$ & $1 / 4$ \\
\hline Bone deformity & $3 / 10$ & $0 / 5$ & $4 / 8$ & $1 / 4$ \\
\hline Gait abnormality & $4 / 10$ & $0 / 5$ & $2 / 8$ & $1 / 4$ \\
\hline Hypocalcemic manifestation & $2 / 10$ & $0 / 5$ & $2 / 8$ & $0 / 4$ \\
\hline Abnormal bone profile & $8 / 10$ & $2 / 5$ & $8 / 8$ & $2 / 4$ \\
\hline \multicolumn{5}{|l|}{ Response to treatment } \\
\hline Yes & $5 / 10$ & $5 / 5$ & $8 / 8$ & $4 / 4$ \\
\hline No & $5 / 10$ & $0 / 5$ & $0 / 8$ & $0 / 4$ \\
\hline \multicolumn{5}{|l|}{ Maintenance therapy } \\
\hline Weekly & $4 / 10$ & $0 / 5$ & $2 / 8$ & $1 / 4$ \\
\hline Twice monthly & $1 / 10$ & $1 / 5$ & $2 / 8$ & $0 / 4$ \\
\hline Monthly & $0 / 10$ & $4 / 5$ & $4 / 8$ & $3 / 4$ \\
\hline $1,25-(\mathrm{OH})_{2}$ vitamin $\mathrm{D}$ & $5 / 10$ & $0 / 5$ & $0 / 8$ & $0 / 3$ \\
\hline $\begin{array}{l}\text { https://ec.bioscientifica.com } \\
\text { https://doi.org/10.1530/EC-21-0102 }\end{array}$ & $\begin{array}{r}\text { ( } 2021 \\
\text { Published by Biosc }\end{array}$ & $\begin{array}{l}\text { hors } \\
\text { a Ltd }\end{array}$ & $\begin{array}{l}\text { This work is licen } \\
\text { Attribution-NonC }\end{array}$ & $\begin{array}{l}\text { der a Creative Commons } \\
\text { rcial-NoDerivatives } 4.0\end{array}$ \\
\hline
\end{tabular}


The ability to understand the different responses to treatment based on the gene mutation and zygosity can direct us to the type of treatment for an individual patient. We noticed a symptomatic and biochemical improvement in all heterozygous patients after treatment with supratherapeutic doses of vitamin D (i.e. 50,000-100,000 IU of vitamin D2 weekly) for 8-12 weeks, regardless of their mutation, and none of them required calcitriol $\left(1,25-[\mathrm{OH}]_{2}\right.$ vitamin D), whereas the homozygous group required more frequent therapy in the form of weekly to twice monthly maintenance high-dose vitamin D therapy, and some of them needed calcitriol for a lifetime. These patients required closer follow-up and renal ultrasonography every year to check for signs of calcifications or nephrolithiasis, which was negative in all patients receiving calcitriol. On a genetic basis, we noticed that all the patients who carried the c.367+1G>A mutation responded to a high dose of vitamin $\mathrm{D}$ therapy and none of them needed to be on calcitriol, compared with the patients who carried the homozygous mutation in c.768dupT, where some of them required calcitriol for their treatment, suggesting a more severe disease phenotype in those patients.

From the above data, it is interesting to highlight that there was no clear genotype/phenotype correlation, with a wide range in disease expression, severity, and response to treatment. Although we did not find any clear explanation for the $27.8 \%$ of homozygous non-responders to a high dose of cholecalciferol, still we can't exclude the possibility of other factors that could contribute to this variability. As some studies reported the effect of Glutathione (a major antioxidant and a cofactor of many enzymes) on the expression of the vitamin $\mathrm{D}$ genes and receptors, which can predispose the body to 25(OH)VD3 deficiency in obese and type 2 diabetic patients $(15,16)$. However, none of our patients were obese and they didn't have diabetes.

A mutation in the CYP2R1 gene leads to a new form of genetic vitamin $\mathrm{D}$ deficiency that can be identified as an entity with semi-dominant inheritance, as all the affected patients showed variable disease manifestations regardless of their homozygous/heterozygous status, except for hypocalcemic manifestations, which were only observed in the homozygous patients. Patients with the CYP2R1 mutations carried a more complex disorder, in which they presented with classical symptoms of vitamin D deficiency and associated rickets, but they required unique management. An important observation in our study, in which patients showed regression after decreasing the vitamin $\mathrm{D}$ dose to a daily requirement dose or discontinuation of the treatment, as well as similarly noticed in non-compliant patients who were treated with calcitriol, was that those patients need to be on maintenance therapy for life.

As we were able to follow our patients throughout their adulthood, we noticed that the patients who were diagnosed and started on treatment earlier in life had milder symptoms and a better outcome than the patients who were managed later in life. Some patients were suffering from bone ache and bone deformities during their childhood, and consequently sought multiple medical advices that led to mismanagement and/or underdiagnosis of the disease. Such missed cases resulted in severe short stature, severe bone pain, and deformities that led to the requirement of a wheelchair. Therefore, we believe that identifying the problem with early diagnosis and early intervention can have a great impact on the overall prognosis of patients with this disease.

We should consider vitamin D-dependent rickets type $1 \mathrm{~B}$ as a diagnosis in (i) all children with early symptoms of vitamin D deficiency who depend on a high dose of vitamin D treatment or are not responding to the high dose of vitamin D therapy with no identifiable organic cause, and (ii) individuals with familial severe vitamin D deficiency. Confirmation of the diagnosis should be pursued by performing molecular analysis of the CYP2R1 gene in the patients and their families. We recommend starting treatment with a supra-therapeutic dose of vitamin D therapy (50,000 IU/week for 8-12 weeks), followed by 50,000 IU maintenance therapy, between weekly and monthly as required. However, starting them on calcitriol in case of no response will result in clinical and biochemical improvement. This is in addition to optimizing the dairy product requirement and adding calcium supplements if needed, aiming to maintain 25-OH vitamin D levels within the normal range and modifying the treatment and the dose based on the clinical and biochemical response. We believe using calcifediol (25-OH-D3), which bypasses the 25-hydroxylase defect, is the ideal treatment for this condition, which was used with significant benefit in some patients (12), but unfortunately, this treatment is not available in our center. Also, adjuvant therapy of L-cysteine (a GSH precursor) along with vitamin D supplements might be beneficial in non-responsive patients, as suggested by some reports $(15,16)$. Therefore, initiation of therapy should be based on genetics and the availability of different types of vitamin D analogs in the individual's healthcare facility. https://ec.bioscientifica.com https://doi.org/10.1530/EC-21-0102 (c) 2021 The authors Published by Bioscientifica Ltd
This work is licensed under a Creative Commons Attribution-NonCommercial-NoDerivatives 4.0 International dicense ifica com at 04/26/2023 09:38:37AM 


\section{Conclusion}

Our data support that CYP2R1 plays a major role in 25-hydroxylation, which is fundamental in the activation of vitamin D. It is suggested that a higher percentage of CYP2R1 mutations-related vitamin D deficiency is found in the Saudi population where there is a high rate of consanguinity. To our knowledge and to date, this is the largest cohort series reporting CYP2R1-related 25-hydroxylase deficiency worldwide and the first study to focus on clinical, biochemical presentation, as well as the management of these patients. We believe that our study will help in the diagnosis, treatment, and prevention of similar cases in the future. Further studies are needed to consider calcifediol use as a treatment of this disease entity and to determine its long-term effect.

\section{Supplementary materials}

This is linked to the online version of the paper at https://doi.org/10.1530/ EC-21-0102.

\section{Declaration of interest}

The authors declare that there is no conflict of interest that could be perceived as prejudicing the impartiality of the research reported.

\section{Funding}

This work was supported by the Research Advisory Council and the project was IRB approved (RAC \# 2151206). And funded by the Pediatric Research Committee at King Faisal Specialist Hospital and Research Centre (Research \# 2190480).

\section{References}

1 Carpenter TO, Shaw NJ, Portale AA, Ward LM, Abrams SA \& Pettifor JM. Rickets. Nature Reviews: Disease Primers 2017317101. (https://doi.org/10.1038/nrdp.2017.101)

2 Abdullah MA, Salhi HS, Bakry LA, Okamoto E, Abomelha AM, Stevens B \& Mousa FM. Adolescent rickets in Saudi Arabia: a rich and sunny country. Journal of Pediatric Endocrinology and Metabolism 2002 15 1017-1025. (https://doi.org/10.1515/jpem.2002.15.7.1017)

3 Al Shaikh AM, Abaalkhail B, Soliman A, Kaddam I, Aseri K, Al Saleh Y, Al Qarni A, Al Shuaibi A, Al Tamimi W \& Mukhtar AM. Prevalence of vitamin D deficiency and calcium homeostasis in Saudi children. Journal of Clinical Research in Pediatric Endocrinology 20168 461-467. (https://doi.org/10.4274/jcrpe.3301)

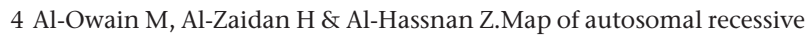
genetic disorders in Saudi Arabia: concepts and future directions. American Journal of Medical Genetics: Part A 2012 158A 2629-2640. (https://doi.org/10.1002/ajmg.a.35551)

5 Miller WL. Genetic disorders of vitamin D biosynthesis and degradation. Journal of Steroid Biochemistry and Molecular Biology 2017 165 101-108. (https://doi.org/10.1016/j.jsbmb.2016.04.001)

6 Zhu JG, Ochalek JT, Kaufmann M, Jones G \& DeLuca HF. CYP2R1 is a major, but not exclusive, contributor to 25-hydroxyvitamin D production in vivo. PNAS $201311015650-15655$. (https://doi. org/10.1073/pnas.1315006110)

7 Cheng JB, Motola DL, Mangelsdorf DJ \& Russell DW. De-orphanization of cytochrome P450 2R1: a microsomal vitamin D 25-hydroxilase. Journal of Biological Chemistry 2003278 38084-38093. (https://doi.org/10.1074/jbc.M307028200)

8 Al Mutair AN, Nasrat GH \& Russell DW. Mutation of the CYP2R1 vitamin D 25-hydroxylase in a saudi arabian family with severe vitamin D deficiency. Journal of Clinical Endocrinology and Metabolism 201297 E2022-E2025. (https://doi.org/10.1210/jc.2012-1340)

9 Casella SJ, Reiner BJ, Chen TC, Holick MF \& Harrison HE. A possible genetic defect in 25-hydroxylation as a cause of rickets. Journal of Pediatrics 1994124 929-932. (https://doi.org/10.1016/s00223476(05)83184-1)

10 Cheng JB, Levine MA, Bell NH, Mangelsdorf DJ \& Russell DW. Genetic evidence that the human CYP2R1 enzyme is a key vitamin D 25-hydroxylase. PNAS 2004101 7711-7715. (https://doi.org/10.1073/ pnas.0402490101)

11 Thacher TD, Fischer PR, Singh RJ, Roizen J \& Levine MA. CYP2R1 mutations impair generation of 25-hydroxyvitamin $\mathrm{D}$ and cause an atypical form of vitamin D deficiency. Journal of Clinical Endocrinology and Metabolism 2015100 E1005-E1013. (https://doi.org/10.1210/ jc.2015-1746)

12 Molin A, Wiedemann A, Demers N, Kaufmann M, Do Cao J, Mainard L, Dousset B, Journeau P, Abeguile G, Coudray N, et al. Vitamin D-dependent rickets type 1B (25-hydroxylase deficiency): a rare condition or a misdiagnosed condition? Journal of Bone and Mineral Research 201732 1893-1899. (https://doi.org/10.1002/jbmr.3181)

13 Holick MF, Binkley NC, Bischoff-Ferrari HA, Gordon CM, Hanley DA, Heaney RP, Murad MH, Weaver CM \& Endocrine Society. Evaluation, treatment, and prevention of vitamin D deficiency: an endocrine society clinical practice guideline. Journal of Clinical Endocrinology and Metabolism 201196 1911-1930. (https://doi.org/10.1210/jc.2011-0385)

14 Thomas L. Parathyroid hormone (PTH). In Clinical laboratory diagnostics: use and assessment of clinical laboratory results, pp. 248-250. Frankfurt, Germany: TH-Books, 1998.

15 Jain SK, Parsanathan R, Achari AE, Kanikarla-Marie P \& Bocchini JA. Glutathione stimulates vitamin D regulatory and glucosemetabolism genes, lowers oxidative stress and inflammation, and Increases 25-hydroxy-vitamin D Levels in blood: a novel approach to treat 25-hydroxyvitamin D deficiency. Antioxidants and Redox Signaling 2018 29 1792-1807. (https://doi.org/10.1089/ars.2017.7462)

16 Parsanathan R \& Jain SK. Glutathione defciency induces epigenetic alterations of vitamin D metabolism genes in the livers of high-fat diet-fed obese mice. Scientific Reports 20199 14784. (https://doi. org/10.1038/s41598-019-51377-5)

Received in final form 20 April 2021

Accepted 16 June 2021

Accepted Manuscript published online 16 June 2021 https://ec.bioscientifica.com https://doi.org/10.1530/EC-21-0102
(C) 2021 The authors Published by Bioscientifica Ltd
This work is licensed under a Creative Commons Attribution-NonCommercial-NoDerivatives 4.0 international License.ifica com at $04 / 26 / 2023 \quad 09: 38: 37 \mathrm{Am}$ 